\title{
VANTAGENS E DIFICULDADES DAS TECNOLOGIAS DE INFORMAÇÃO E COMUNICAÇÃO NA EDUCAÇÃO
}

\author{
Luanna Aretuska Silva Santos ${ }^{1}$
}

RESUMO: O presente estudo busca demonstrar de forma clara e concisa as dificuldades do uso das tecnologias de informação e comunicação, bem como, suas vantagens no âmbito educacional. Trata-se de um estudo descritivo, desenvolvido através de pesquisa bibliográfica, com base nos artigos científicos disponíveis em quatro bases de dados (Google Acadêmico, Research Gate, Scientific Eletronic Library Online (SCIELO) e sites educacionais), com uma abordagem qualitativa. Foram selecionados artigos científicos, publicados entre os anos de 2012 a 2020. Para a realização da revisão bibliográfica foram selecionados 35 artigos que apresentavam maior relevância e dentro dos critérios de inclusão. À vista disso, o trabalho ratifica que as TICs veem remodelando a esfera educacional e quebrando paradigmas e conceitos do modelo tradicional de ensino, porém ainda existem algumas dificuldades relacionadas a essa temática.

Palavras-chave: Tecnologia de Informação e Comunicação. Dificuldades das TICs. TICs na educação.

ABSTRACT: The present study seeks to demonstrate clearly and concisely the difficulties of using information and communication technologies, as well as its advantages in the educational field. This is a descriptive study, developed through bibliographic research, based on scientific articles available in four databases (Google Scholar, Research Gate, Scientific Electronic Library Online (SCIELO) and educational sites), with a qualitative approach. Scientific articles were selected, published between the years 2012 to 2020. To carry out the literature review, 35 articles were selected that were more relevant and within the inclusion criteria. In view of this, the work confirms that ICTs are remodeling the educational sphere and breaking paradigms and concepts of the traditional teaching model, but there are still some difficulties related to this theme.

Keywords: Information and Communication Technology. Difficulties of ICTs. ICTs in education.

\section{INTRODUÇÃO}

Atualmente com a nova era digital, os alunos desenvolvem habilidades no manuseio com muita facilidade de qualquer aparelho tecnológico como celular, computador e internet. Esses recursos são denominados TICs (Tecnologia da Informação e Comunicação). Assim, a tecnologia está no dia a dia, não só do aluno, mas também dos

\footnotetext{
${ }^{I}$ Bacharel em Ciências biológicas pela Universidade Tiradentes (Unit-SE), 2020. Docente em Formação Pedagógica para não Licenciados em Biologia pela Universidade Cruzeiro do Sul. Email: aretuskabioo8@gmail.com.
} 
professores, que utilizam como metodologias jogos, vídeos, imagens e outros recursos em suas aulas (ZANELLA et al., 2017).

As TICs surgiram como uma forma de auxiliar, facilitar e agregar diversas áreas, como a educação, principalmente no quesito de introduzir os computadores no cenário escolar, para trazer mais agilidade no acesso à informação. Este mesmo recurso possibilitou uma nova didática no processo de ensino-aprendizagem, tanto para o professor quanto para o aluno. Essas tecnologias dentro da educação permitem ampliar o conhecimento e inserir novidades no contexto escolar (MARTINES et al., 2018).

As tecnologias oferecem diversas vantagens no âmbito educacional, que possibilita ampliação na busca por conhecimentos e contribuindo para o progresso de ensino e aprendizagem. Além de ser de grande interesse para o aprimoramento da melhoria no sistema educacional (JARDIM et al., 2018). No entanto, incorporar essas tecnologias na educação não é uma tarefa fácil, requer vários fatores, na qual destaca-se: gastos públicos, infraestrutura das escolas e domínio dos professores com os novos recursos tecnológicos (FERNANDES; OLIVEIRA; ALBUQUERQUE, 2020).

Mesmo com muitos professores sem capacitação para essa nova fase na educação, a 207 maioria reconhece a relevância das (Tecnologias de Informação e Comunicação) as TICs, como auxiliadora no processo de ensino- aprendizagem. Desta maneira, a tecnologia se torna uma aliada ao processo e que está se tornando cada vez mais indispensável na educação, pois ela consegue unir o professor com o aluno, através de aulas mais atrativas e dinâmicas, o que se faz necessário utilizar esse recurso a favor da educação (SANTOS; MACEDO; CHAGAS, 2017).

Diversas áreas podem utilizar as TICs como uma facilitadora de ensino, a exemplo da biologia, que é uma das matérias consideradas mais difíceis no conteúdo programático de ensino, e dentro dela existe uma área voltada para o estudo dos vegetais: a botânica, que é vista como uma área complexa e fora da realidade do aluno, o que dificulta o entendimento sobre o assunto (DUTRA; GÜLLICH, 2016).

Outra questão é que seu ensino está muito ligado a métodos convencionais, ou seja, limitada a exercícios de livros, aulas em sua maioria expositivas, no qual o aluno muitas vezes não aprende, apenas decora o conteúdo para realizar as atividades avaliativas e com 
termos bem difíceis. Assim, o aluno não se sente estimulado, interessado e não traz quase sempre um resultado proveitoso na aprendizagem do mesmo (AVELINO et al., 2019).

Diante do exposto, se torna necessário novas metodologias de ensino, que estimula o aluno a se interessar pelo conteúdo, e que o professor possa instigar seus alunos, com o uso das tecnologias, implementando o conteúdo dos livros, com imagens, vídeos, audiovisuais, jogos, para atrair o interesse do aluno (MACHADO; POLETTO; ALVES, 2019).

Desta forma, este trabalho tem como objetivo mostrar como o uso das tecnologias de informação e comunicação podem contribuir no auxílio do ensino de botânica.

\section{METODOLOGIA}

O presente trabalho consiste em uma revisão bibliográfica de caráter descritivo, que as etapas adotadas nesta revisão foram: definição do objetivo, da pergunta de pesquisa, de banco de dados, dos critérios de inclusão e exclusão, das palavras-chave; seleção de trabalhos; análise e síntese dos estudos incluídos na revisão de literatura; redação, desenvolvimento e conclusão dos resultados. Foi realizado entre o período de agosto a novembro de 2020, com a seleção das publicações (artigos científicos, atas de conferências, teses de doutorado, dissertações de mestrado, trabalhos de conclusões de curso).

Foi utilizado como fontes de pesquisa as seguintes bases de dados: Google Acadêmico, Research Gate, Scientific Eletronic Library Online (SCIELO), e sites educacionais, com o uso de palavras chaves: "Tecnologia de Informação e Comunicação", “Tecnologias e Educação”, "Dificuldade e tecnologia e educação”, “TICs na educação”.

O critério utilizado para a inclusão baseou-se em selecionar trabalhos e pesquisas publicados nos últimos oito anos, com os descritos mencionados anteriormente, como também temáticas englobando a educação associada as tecnologias de comunicação e informação. Para critério de exclusão, os artigos que não se encaixava com a temática abordada, e ou com conteúdo considerados relativamente superficiais e que não possuía os descritores acima mencionados como inclusão na pesquisa. 
Por se tratar de uma pesquisa de revisão de literatura, não foi necessário submeter a pesquisa ao Comitê de Ética e Pesquisa da instituição. Todavia, os pesquisadores se comprometem com os direitos autorais dos artigos utilizados.

\section{RESULTADO E DISCUSSÃO}

Foram selecionados um total de 35 artigos científicos, obtidos através das bases de dados e que disponibilizavam de maior interesse de relevância e que estão dentro dos critérios de inclusão e com publicações nos períodos entre 2012 a 2020.

\section{I Tecnologia de informação e comunicação}

O desenvolvimento das tecnologias de informação e comunicação influenciam a sociedade de maneira em geral, porém, de modo particular, a "velocidade de propagação da informação”. Em muitas situações, principalmente as de crises, foram solucionadas através de pessoas que detinham informações e conhecimentos (CASTILHO, 2015).

A população está inserida dentro de uma rotina em que se utiliza tecnologias desde o acordar até o dormir, e nas mais diversas ocasiões do dia. Desde o início ao utilizar o despertador, até a solicitação de um carro por aplicativo, nas transferências bancárias, ao assistir uma televisão, fazer uma ligação, e até mesmo interagir com a família, amigos e no trabalho (NASCIMENTO; FARIAS; MARTINS, 2018).

Nesses e em outros momentos, faz se o uso de recursos midiáticos, que podem ser sistemas operacionais, plataformas virtuais, aplicativos e programas. Independente do meio, a utilização desses recursos tornou-se imprescindível no nosso cotidiano (MEC, 2020).

CRUZ (2020), evidencia que à medida que o tempo passa, o mundo vai progredindo muito no quesito da utilização das TICs, que tem ocupado várias áreas da sociedade, que perpassa por áreas da cultura, política, economia, história, arte, música, comunicação e como também na educação.

Como destaca Reis et al., (2018)

A utilização das TIC em sala de aula possibilita aos alunos um determinado nível de conhecimento que os leva a novas práticas sociais em contextos específicos. Assim, hoje, os alunos precisam estar sintonizados com as novas tecnologias da 
informação e comunicação e mais do que apenas saberem fazer uso da internet e dos seus espaços, é preciso, antes de tudo, que os alunos saibam construir o conhecimento a partir dela.

Silva et al. (2020), ressalta que as mídias e os meios digitais espalharam-se pelo mundo, tornando a população cada vez mais dependentes dela, e é inegável e indiscutível que as tecnologias adentraram a esfera educacional, e é devido a essa ferramenta que os alunos e professores conseguem ter acesso mais rápido as informações, em comparação com as gerações anteriores.

Segundo Campos (2013), o aparecimento dos meios tecnológicos é determinado por meio da cobertura mundial, ou seja, através do alcance que ela pode abranger e também, por conta da comunicação e interação de todos os recursos tecnológicos, que mudou, está mudando e irá mudar a todo momento a história da humanidade. Castilho (2015), ainda reforça que a utilização das TICs é um sistema inconvertível e que ao explorar as práticas de ensino recentes, essa tecnologia pode contribuir para formação do homem.

\subsection{Tecnologia na educação}

A partir da descoberta dos computadores e sua introdução nas escolas de diversos países na década de 70, o seu uso na educação passou a ser uma ferramenta de suma importância para este meio. Juntamente com os computadores, houve a inserção de scanners, impressoras e drives e até mesmos das câmeras fotográficas digitais. Tal agrupamento tecnológico, foi denominado como tecnologia da informação, ou TI, como é comumente falado (BARROS; GARCIA; MEDEIROS, 2018).

Com o aparecimento da internet, outras funções foram incorporadas nas escolas. A introdução dos computadores e programas como o Word, e-mail e_ferramentas de busca, foram nomeados como TICs, tecnologia da informação e comunicação, que se refere a diversidade de equipamentos e suas funções, que permite o usuário, receber, armazenar, criar, capturar e transmitir informações (ROZA, 2018).

As TICs possibilitam uma nova alternativa de adquirir conhecimento, o que faz com que o aluno crie sua própria maneira de aprendizagem como também, instigue a procura por informações. Uma das principais funções das TICs é seu uso como 
facilitadora, tanto no processo de ensino como no de aprendizagem e tem como base, unir a vivência dos alunos com os assuntos lecionados em sala (MATOS et al., 2019).

A Unesco (2017), menciona as vantagens das TICs na sociedade como:

As TICs podem contribuir para o acesso universal à educação, a equidade na educação, a qualidade de ensino e aprendizagem, o desenvolvimento profissional de professores, bem como melhorar a gestão, a governança e a gestão educacional ao fornecer a combinação certa e organizada de políticas, tecnologias e capacidades.

Andrade e Ferrete (2018), enfatiza que além das tecnologias facilitar o acesso as informações dos alunos, o compartilhamento dessas informações acontece de forma muito rápida e significativa, tornando esse mecanismo essencialmente importante no contexto educacional.

A associação das TICs no processo de ensino-aprendizagem permite agregar novas metodologias no uso da leitura e da escrita, tanto no individual, quanto no social. Nesta perspectiva, o aluno lê através de recursos e meios digitais e tecnológicos, e desta forma a interação acontece por meio dos cenários, compostos por imagens, sons e textos que estão englobados como sistemas de aprendizagem comunicativa (POSSOLLI; MARCHIORATO; NASCIMENTO, 2018).

A produção que envolve sons, imagens, elementos em movimento, cenários coloridos tendem a seduzir mais, pois esses elementos ativam o lado racional, afetivo, abstrato (MEC, 2020). Em consonância, Moran (2013), cita que a ligação com os meios eletrônicos é bastante prazerosa, pois não é imposta, ela acontece de forma natural, acontece por meio da sedução, da emoção, do sensorial.

Almeida (2020), salienta que as TICs jamais irão substituir o quadro, o giz e o caderno. A ideia principal é aprimorar novas técnicas de ensino e ao mesmo tempo acompanhar o avanço tecnológico, mesmo dentro de uma sala de aula. E tendo em vista que, a atual sociedade é a mais tecnológica e que consequentemente os alunos também, é necessário que o professor procure por meios que aumenta o entusiasmo dos alunos e a permanência na escola. Andrade e Ferrete (2019), ainda ressalta que o modo de se comunicar e aprender mudou e que o de ensino também necessita mudar.

E um contraponto bastante importante destacar, é que existem inúmeras possibilidades de se utilizar as TICs em sala de aula, e que, se usado com criatividade e 
inovação, as aulas não serão as mesmas todos os dias, pelas diversas possibilidades de dinamizar as metodologias (NASCIMENTO; FARIAS; MARTINS, 2018). E além disso, a reunião que as TICs proporcionam em meio virtual, é um reflexo do que já ocorre no cotidiano, visto que, o ser humano sempre fez parte de vários grupos e que sempre teve o hábito de se reunir para estudar, trabalhar, se divertir e as mais variadas ocasiões (BOZZA, 2016).

\subsection{Dificuldades das TICs}

Vários obstáculos dificultam a introdução dessas tecnologias no sistema educacional. A primeira delas é a falta de incentivo financeiro do Governo. No entanto, em 1997, foi criado pelo Ministério da Educação, um Programa Nacional de Tecnologia Educacional (ProInfo) que tem como intuito promover o uso da tecnologia como ferramenta de enriquecimento pedagógico no ensino público fundamental e médio (FNDE, 2017).

Porém, em 2007, houve um decreto no qual estabeleceu um novo objetivo para o programa, que consiste em promover o uso pedagógico das tecnologias de Informação e Comunicação nas redes públicas de educação básica. Este programa leva para as escolas, computadores, recursos digitais e conteúdos educacionais. Todavia, os estados e municípios devem assegurar a estrutura das escolas para receber essas ferramentas e capacitar seus profissionais para o uso dessas tecnologias (MEC, 2018).

Silva; Prates e Ribeiro (2016), enfatiza a precariedade da infraestrutura de várias escolas para suportar essas tecnologias, sendo assim um empecilho do uso das TICs na educação. Cabe ressaltar que, a introdução dessas tecnologias nas escolas não assegura a aprendizagem, pois, muitas escolas dispõem dessas tecnologias, porém, na maioria das vezes são utilizadas de forma incorreta, servindo apenas como uma mera ferramenta auxiliadora de funções básicas como: imprimir provas ou escanear documentos.

Já Medeiros e Galiazzi (2018), corroboram a falta de domínio de alguns professores, que se sentem incapacitados para manusear as tecnologias, principalmente porque, em alguns casos, os professores não tiveram uma experiência na graduação com matérias referentes a tecnologia. 
Segundo a pesquisa "TIC Educação", 39\% dos professores das escolas públicas e privadas tiveram alguma relação ou disciplina na graduação referente ao uso das tecnologias em sala de aula, ou seja, mais da metade de professores formados não tiveram nenhum contato direto com esses recursos na sua formação (AGÊNCIA BRASIL, 2020).

A falta de tempo dos professores, a resistência a mudanças, que pode ser provocada pela insegurança e a pouca confiança na utilização das TICs, são uns dos grandes dilemas enfrentados pelos professores perante as tecnologias. Assim, observa-se necessário não somente apoiar os professores nessa transição, mas como também, fornece uma formação adequada a integração pedagógica das TICs (RODRIGUES, 2014).

Porém, basta estar mais atento para sala de aula e perceber que a maioria dos alunos passam mais tempo conectados com o mundo virtual do que em contato com os colegas e professores, e isso se dá por conta que os alunos já nascem conectando incontáveis dispositivos técnicos. Por exemplo, uma criança com aproximadamente 3 a 4 anos já manuseou alguma função em smartphones, tablets entre outros (MOREIRA; LUDOVICO; KAROLESKY, 2018).

Isso porque, antes mesmo de uma criança ingressar à escola, ela passa por dois processos educacionais de suma importância: o familiar e a mídia eletrônica. O familiar, é o ambiente onde a criança desenvolve suas habilidades cerebrais, emocionais e de linguagens. Já pela mídia eletrônica, a criança começa desde cedo a ser influenciada pelo principal meio de comunicação e informação: a televisão. Com ela, a criança conhece vários estilos de vidas, pessoas diferentes, aprende as criar coisas, estimula um misto de sensações, etc. (MORAN, 2013).

Desta maneira, o contato direto com uma ferramenta de informação e comunicação, estimula desde cedo nas crianças, uma visão ampliada e desperta uma curiosidade sobre os meios tecnológicos como: computadores, tablets, celulares, games. Por isso, são denominados como "nativos digitais", que são aqueles que não se contentam apenas em ler manuais, eles vão e fazem, ou seja, aprende fazendo. Que teoricamente já é o que se faz normalmente quando eles aprendem sozinhos a manusear um aplicativo, um jogo ou algo relacionado (TOLOMEI, 2017). 
Esse novo contexto educativo está inserido no mundo moderno, e isso impõe um novo posicionamento das escolas. As escolas contribuem para o desenvolvimento social e tecnológico dos alunos, e isso está assegurado pelo estatuto da Criança e do Adolescente (ECA), que declara que as crianças e os adolescentes possuem o direito a uma educação de qualidade, e que neste meio está inclusa a introdução da tecnologia, que neste atual cenário, é uma ferramenta de extrema importância para a formação cidadã (AZEVEDO et al., 2014).

Entretanto, nem todas as escolas usufruem do benefício de ter acesso a internet, e quando dispõem é de baixa qualidade o que acarreta dificuldades no uso das TICs dentro das escolas. Além do ambiente escolar, a maioria dos alunos, principalmente os de escola pública, não possuem nem aparelhos tecnológicos conectados à internet (STINGHEN, 2017).

\section{CONSIDERAÇÕES FINAIS}

Em virtudes dos fatos mencionados, é notório que existem inúmeros problemas relacionados as tecnologias de informação e comunicação (TICs) na educação como: Governo, infraestrutura das escolas, disponibilidades de aparelhos, principalmente com acesso à internet, insegurança dos professores, ocasionado pela formação acadêmica restrita. Porém, esse trabalho de revisão mostra que as TICs vêm remodelando a esfera educacional e quebrando paradigmas e conceitos do modelo tradicional de ensino, favorecendo uma troca recíproca de informações entre professores e alunos. Desta maneira, mostra-se a importância do uso de tecnologias de informação e comunicação para o ensino educacional, na qual permite aos alunos uma experiência mais real e motivadora dos assuntos abordados

\section{REFERÊNCIAS}

ALMEIDA, C. F. B. A inserção das TICs como ferramenta facilitadora do processo de ensino-aprendizagem: contribuição para língua inglesa. Diversitas Journal, v. 5, n. 2, abr./jun. 2020, p. IIIO-II2O. 
ANDRADE, L. G. S. B.; FERRETE, R. B. Metodologias ativas e a educação profissional e tecnológica: invertendo a sala de aula em vista de uma aprendizagem significativa. Revista Educação Profissional e Tecnológica, v. 3, n. 2, 2019, p. 86-98.

AVELINO, F. M.; AVELINO, C. M.; SILVA, L. C. M.; FERREIRA, J. G. C.; LIMA, M. M. o. Jogo didático como proposta no ensino de botânica: desenvolvendo metodologia inovadora com alunos de uma escola estadual de Floriano (PI). International Jornal Educacional and Teaching- PDVL, Recife, v. 2, n. 3, set.-/dez, 2019, p. I - I4.

AZEVEDO, A.; BAFFA, A. M.; RAMOS, A. C. P.; PINHEIRO, A. L.; ALMEIDA, D.; OSTLER, D. A.; MENDONÇA, G. B. A.; MUNARI, K. B.; MOZZER, L. D.; PÉRICO, L. A. S.; MAGAlHÃES, M. V. C.; FERREIRA, P. V.; GIANNOTTI, R. C. B.; SARAGIOTO, V. A. V. TICs na Educação: multivisões e reflexões coletivas. Revista Educação \& Linguagem, v. 17, n. 2, jul./dez, 2014, p. 215-236.

BARROS, M. H. S.; GARCIA, P. H. M.; MEDEIROS, G. N. O uso das TIC's no ensino de geografia: Aproximação entre conteúdos e técnicas. Revista Ensin@-UFMS-Ensino de Geografia e áreas afins, v.I, n.3, 2018.

BOZZA, T. C. L. O uso da tecnologia nos tempos atuais: Análise de programas de intervenção escolar na prevenção e redução da agressão virtual. Dissertação de Mestrado, Universidade Estadual de Campinas, 2016

CAMPOS, G. A. Educação na Cibercultura: paradigmas educacionais emergentes da revolução digital. Jornal Alcar, v. 2, n. II, 2013.

CASTILHO, L. B. O uso da tecnologia da informação e comunicação (TIC) no processo de ensino e aprendizagem em cursos superiores. UNIVERSIDADE FUMEC, v. 4, n. 2, 2015 .

CRUZ, M. L. O. B. As tics no uso da linguagem e aprendizagem de línguas. Open Minds International Journal. São Paulo, v. I, n. 2, 2020, p. 15- 24.

DUTRA, A. P.; GÜLLICH, R. I. C. Ensino de botânica: metodologias, concepções de ensino e currículo. Revista Ensino de Ciências e Tecnologia, V. 6, n. 2, jul./dez. 2016, p. 3953.

FERNANDES, V. B., OLIVEIRA, M. S. C.; ALBUQUERQUE, H. S. Desenvolvimento do aplicativo funções químicas quiz como ferramenta de ensino-aprendizagem aplicado às aulas de química. Brazilian Journal of Development, Curitiba, v. 6, n. 8, 2020, p. 5731457323 .

JARDIM, R. R.; PEREIRA, D. S.; ROCHA, P. S.; LIMA, J. V.; FILHO, A. B. C. Avaliação do impacto prático dos saberes adquiridos em termos de introdução das TICs na Educação Básica com base no modelo SAMR, VII Congresso Brasileiro de Informática na Educação, 2018. 
JÚNIOR, M. C. Baixa velocidade e falta de capacitação dificultam o uso da internet nas 2020. Disponível em: <https://agenciabrasil.ebc.com.br/educacao/noticia/2016-09/baixa-elocidade-e-falta-decapacitacao-dificultam-uso-da-internet-em>.Acesso em: 23/o9/2020.

MACHADO, T. A.; POLETTO, R. S.; ALVES, D. S. Ensino de botânica e atualização de conhecimentos científicos para o ensino superior: uma revisão sistemática da literatura. Revista Ensino de Ciências e Tecnologia, v. 9, n. 2, mai./ago. 2019, p.93-107.

MARTINES, R. S.; MEDEIROS, L. M.; SILVA, J. P. M.; CAMILLO, C. M. O uso das TICs como recurso pedagógico em sala de aula. Congresso Internacional de Educação e Tecnologias, 2018.

MATOS, J. D. V.; SILVA, J. R. C.; RIBEIRO, A. F. S.; GOMES, R. M. M.; FERREIRA, J. C.; MATOS, F. B. Aprendizagem Significativa por meio do Uso de TICs: Levantamento das Produções da Área de Ensino de 2016 a 2018. Novas Tecnologias na Educação, v. 17, n. I, 2019, p.466-475.

MEDEIROS, A. L. S.; GALIAZZI, M. C. Especificidades sobre formação de professores no encontro brasileiro de ensino superior à distância (ESUD). Revista de Ciências Humanas, v.19, n.2, 2018, p.52-67.

MORAN, J. M.; MASETTO, M. T.; BEHRENS, M. A. Novas Tecnologias e Mediação Pedagógica. 21 İ $^{\mathrm{a}}$ Edição. Editora Papirus. p.36-46, 2013.

MOREIRA, J. C. G.; LUDOVICO, F. M..; KAROLESKY, M. L. As Metodologias Ativas e Seus Atores Aprendentes. Revista Pleidae, v. 12, n.25, dez., 2018, p. 18-28.

NASCIMENTO, J. L.; FEITOSA, R. A. Metodologias ativas, com foco nos processos de ensino e aprendizagem. Research, Society and Development, v. 9, n. 9, 2020, p. I-17.

NASCIMENTO, V. F.; FARIAS, I. M. S.; MARTINS, A. S. Tecnologias da informação e comunicação na escola: apontamentos sobre uma experiência de formação. Revista educação \& tecnologia, v.23, n.3, 2018.

POSSOLLI, G. E.; MARCHIORATO, A. L.; NASCIMENTO, G. L. Gamificação como recurso educacional na área da saúde: uma revisão integrativa. Revista educação \& tecnologia, v.23, n.3, 2018.

REIS, A. R. S.; NOBREGA, C. T.; DANTAS, D. N.; BARROSO, M. R. O uso das tecnologias da informação e comunicação na educação profissional e tecnológica. Revista educação \& tecnologia, v.23, n.3, 2018. 
RODRIGUES, A. L. Dificuldades, Constrangimentos e Desafios na integração das tecnologias digitais no processo de formação de professores. III Congresso Internacional das TIC na Educação, 2014.

ROZA, R. H. CIÊNCIA DA INFORMAÇÃO, TECNOLOGIA E SOCIEDADE. Revista do Instituto de Ciências Humanas e da Informação, v.32, n.2, jul./dez, 2018, p.177I90.

SANTOS, A. Q.; MACEDO, G. E. L.; CHAGAS, R. J. A botânica na concepção de professores de ciências do ensino fundamental e as dificuldades enfrentadas para ensiná-la. IV Congresso Nacional da Educação, 2017.

SILVA, I. C. S.; PRATES, T. S.; RIBEIRO, L. F. S. As Novas Tecnologias e aprendizagem: desafios enfrentados pelo professor na sala de aula. Revista Em Debate (UFSC), Florianópolis, v. 16, 2016, p. 107-123.

SILVA, Q. P.; LACERDA, M, G. A.; OLIVEIRA, A. A.; RENÔR, R. R. C.; BEZERRA, R. R. M.; LIMA, J. F. S.; SENA, L. S. B.; MONTEIRO, B. V. B. Tecnologias da Informação e Comunicação (TICs) no auxílio do ensino-aprendizagem da Histologia Revisão de literatura. Society and Development, v. 9, n. 7, 2020, p.I-I7.

SOBRE O PROINFO. FUNDO NACIONAL DE DESENVOLVIMENTO da EDUCAÇÃO, 2017. $\quad$ Disponível em: $<$ https://www.fnde.gov.br/programas/proinfo/sobre-o-plano-ou-programa/sobre-oproinfo>. Acesso: Io/o9/2020.

STINGHEN, R, S. Curso de especialização educação na cultura digital. Trabalho de Conclusão de Curso, UNIVERSIDADE FEDERAL DE SANTA CATARINA, 2017.

TIC na educação do Brasil. UNESCO, 2017. Disponível:< https://pt.unesco.org/fieldoffice/brasilia/expertise/ict-education-brazil>.Acesso: Io/og/2020.

TICs 2020. Ministério da Educação, 2020. Disponível em:< https://avamec.mec.gov.br/\#/instituicao/capes/curso/840I/unidade/4704/acessar?contin ue $=$ false $>$. Acesso: $16 /$ ro/2020.

TOLOMEI, B. V. A gamificação como Estratégia de Engajamento e Motivação na Educação. Revista Científica em Educação a Distância, v.7. n.2, 2017 p. $145^{-156 . ~}$

ZANELLA, B. R. D.; LIMA, M. F. W. Refletindo sobre os Fatores de Resistência no Uso das TICs nos Ambientes Escolares, SCIENTIA CUM INDUSTRIA, v. 5, n. 2, 2017, p. 78 -89 . 